\title{
Research of Road Pricing System Based on P System
}

\author{
Yanhua Zhong ${ }^{1,}$ Wei Wei ${ }^{2}$ and Zhiyong Liu ${ }^{1 *}$ \\ ${ }^{1}$ Jiangmen Polytechnic, Jiangmen, Guangdong 529020, China \\ ${ }^{2}$.Xi'an University of technology, Xi'an 710048, China. \\ 12hflowers@163.com,2weiwei@xaut.edu.cn \\ ${ }^{1 *}$ Corresponding Author:jmzy_liu@126.com
}

\begin{abstract}
For urban traffic congestion problem, a communication $P$ systems using parallel computing principles congested road-use pricing model, considering congestion charges in the case, the establishment of travelers travel decision algorithm, travelers dynamic evolution in traffic $P$ system, External charging parameters can be adjusted to influence traveler decisions, thereby affecting the whole system evolution. The congestion pricing model based on P system (CPS_DTAM) of the simulation experiment shows that the algorithm is feasible. This model and particle swarm optimization congestion pricing model (PSO_DTAM) were compared based on the same parameters; the results show that this pricing model taking into accounts the dynamic decision travelers more in line with the real situation.
\end{abstract}

Keywords: intelligent transportation, road pricing, traveler decision-making, communication P system

\section{Introduction}

In recent years, the rapid growth of Chinese private car ownership, resulting in some sections and even some four-tier cities in Commuter peak also appeared traffic congestion [1]. Extending the road infrastructure investment is not only large investment but also long period. Hence the need to change ideas, seek other possible ways to alleviate traffic congestion [2]. If the city section of roads severely congested at certain times, consider using congested roads through toll (congested road-use pricing) for the purpose of easing the congestion. Congestion costs of road use by charging a fee for road users, so that some of these costs do not want to change travelers' travel time or change the itinerary [3]. Each traveler will only consider their own convenience, or consider the economic and time costs they pay. When the traffic smooth, these acts do not affect other travelers, but when road access to reach the limit, it will affect other travelers. Whether it can ease traffic congestion after the implementation of road use charges or how much to charge prices resorted Travelers travel time or travel route change, these need to be modeled by simulation. The scholars of different disciplines analyzed road congestion problems from various angles. Rosenthal [4] from the non-cooperative game theory analysis shows that the effect of the existence of the Nash equilibrium solution for congestion; Levinson [5] study the condition of traffic congestion with a non-cooperative game theory from the perspective of the micro-level analysis of urban traffic congestion, finding that the congestion charging as a cooperative mechanism determine the total cost minimization of travel; Zhang Yi-Mei, et al., [6] studied the mechanism of urban traffic congestion using economic theory and methods; Xu Wei, et al., [7] use of game theory in the "tragedy of the commons" theoretical model of car ownership increases and urban road network capacity for system analysis, explained the roots of traffic congestion generated; Liu ZhiGang, et al., [8] studied the problem of urban traffic congestion with game analysis 
"prisoner's dilemma" problem; Wu Bing, et al., [9] think traffic travel is the game process of derived demand, respectively, with evolutionary game theory to analyze the elastic and inelastic demand for travel conditions. From the perspective of urban cooperative game road network congestion problems are analyzed by Ceng Ying [10]; He Nan, et al., discussed the road construction would expand the induced traffic [11].In reference [12], the authors applied PSO and dynamic traffic assignment theory, dynamic congestion pricing system algorithm strategy, but they did not consider the case of travelers changed the travel route, which does not comply with the real situation. Congested road-use charges are not eager to make some rush hour travel time go by changing the travel time or travel are reluctant to change after a road congestion charge.

The following will be considered in terms of both policy-making of travelers and traffic circulation, the dynamic evolution model of congestion toll road was established by using the principle of $\mathrm{P}$ communication system and the simulation experiments was be done, the simulation results with the literature [12] proposed using particle swarm optimization for dynamic congestion pricing policy model (PSO_DTAM) were analyzed to reveal the changes of traffic flow at different times and in different sections when charges.

\section{Communications P System Optimization Algorithms}

\subsection{Traveler Decision-making Selection Algorithm}

Travelers 'decisions are based on the following idea: the traveler will make decisions based on congestion charging indicators, these indicators variable of congestion charging will bring positive tendency or negative tendencies when the congestion charging indicator variables exceeds a certain critical threshold, the individual make a "yes" or "no" decision. There are three problems need to be resolved.

(1) How to express the consolidated tendency?

(2) How to select the threshold charges?

(3) How to calculate the probability of an individual decision?

Which, there is a latent variable $y_{i}^{*}$, which is a continuous function, and cannot be observed. Indicator variables $x$ and $y_{i}^{*}$ have a linear relationship is expressed as follows:

$$
y_{i}^{*}=\beta_{1}+\cdots+\beta_{k} x_{k i}=\beta^{\prime} x_{i}+u_{i},(k=1 \cdots k)
$$

In equation (1), ${ }^{u_{i}}$ is a random disturbance term, it is meant herein road congestion charging indicator, the formula (1), also known as latent regression equation, which

$$
x_{i}=\left(\begin{array}{c}
1 \\
\vdots \\
x_{k i}
\end{array}\right) \quad \beta=\left(\begin{array}{c}
\beta_{1} \\
\vdots \\
\beta_{k}
\end{array}\right)
$$

Now we consider an individual threshold for deciding whether to travel and set it to 0 . Thus, $\mathrm{x}$ and $\mathrm{y}$ have the following relationship: If $y_{i}^{*}>0$ the travelers make a "yes" decision, otherwise $y_{i}^{*} \leq 0$ make a "No" decision.

$$
y_{i}=\left\{\begin{array}{l}
1, y_{i}^{*}>0 \\
0, y_{i}^{*} \leq 0
\end{array}\right.
$$

If the distribution of random error $u_{i} \sim F(\bullet)$ can be given, then the probability of travelers' decision-making can be determined. Derived as follows: 


$$
\begin{gathered}
P\left(y_{i}=1\right)=P\left(y_{i}^{*}>0\right)=P\left(u_{i}^{*}>-x_{i} \beta\right)=1-F\left(-\beta^{\prime} x_{i}\right) \\
P\left(y_{i}=0\right)=P\left(y_{i}^{*} \leq 0\right)=P\left(u_{i}^{*} \leq-x_{i} \beta\right)=F\left(-\beta^{\prime} x_{i}\right)
\end{gathered}
$$

\subsection{Communication P Systems}

In 1998, scientists in Romania Gheorghe Paun in Finland "Turku Center for AC Research Report Computer Science" was first put forward the idea of membrane computing, and in 2000 published "Computing with membranes" [13]. Subsequently, the membrane computing arouse the research interest of scholars. Beginning in 2003, membrane computing symposium held once a year. Membrane Computing (also called $\mathrm{P}$ systems, MC) with distributed computing and parallel computing features, it is the mechanism through simulation of biological cells to simulate evolution. Membrane computing is as similar as cell hierarchy Nature [14]. Membrane computing combined with other optimization algorithms will get better optimization results. For example, Huang Liang, et al., combining genetic algorithms and membrane computing, shrink and expand in the search area [15]. Pan Lin-Qiang use activities membrane architecture for solving 0-1 knapsack problem [16]. Nishida divided the problem space into many membranes (area) in solving the TSP problem, Tabu Search is used in some areas and Genetic algorithm is used in other areas, and compared with the simulated annealing algorithm, obtained better results [17].

$\mathrm{P}$ systems with symport/antiport are one of the most studied models in membrane computing. Their simple and elegant way to operate has caught the interest of several researchers and many papers have been written on this model or inspired by the operations. Theoretical devices in membrane computing, P systems with symport/antiport consist of a set of nested membranes defining compartments. The nesting present in $\mathrm{P}$ systems with symport/antiport is hierarchical and it defines a cell-tree: a compartment can be contained in at most one compartment and it can contain several compartments. Within each compartments there may be objects evolving and moving to neighbouring membranes following rules specified for the particular system.

In P systems with symport/antiport, as introduced in Păun A., Păun Gh. (2002), computation is restricted to the synchronous movement of objects from one compartment into another. This means that the system contains unstructured objects are not rewritten or changed in any other way. A configuration of the system is given by a finite multiset for each of the compartments; every compartment contains a finite number of objects, whereas the objects initially present in the environment are assumed to have infinite (unbounded) supply. Rules associated to the compartments and inspired by the protein mediated transport described in the previous section, are of one of the following forms.

Formal communication $P$ system as follows:

$$
\Pi=\left(O, E, \mu, w_{1}, \cdots w_{m},\left(R_{1}, \rho_{1}\right), \cdots,\left(R_{m}, \rho_{m}\right), i_{0}\right)
$$

Where

(i) $O$ is an alphabet of objects.

(ii) $E \subseteq O$ (the output alphabet).

(iii) $\mu$ is a membrane structure with m membranes and the regions labeled by the elements of a given set $H=\{1,2, \cdots, m\} . m$ is called the degree of $\Pi$.

(iv) $W_{i} \in O^{*}, 1 \leq i \leq m$ are strings which represent multisets over $O^{*}, O^{*}$ is an arbitrary collection of strings which composed by the character set $O$.

(v) $i_{0}\left(1 \leq i_{0} \leq n\right)$ is the output membrane.

The $n+1$-tuple $\left(\mu, w_{i}, \cdots, w_{n}\right)$ to represent the initial state of implementation of $\mathrm{P}$ systems, communication $\mathrm{P}$ systems are as follows: Within the communication $\mathrm{P}$ system 
cannot produce a new object, the new object can only be come from the environment, which represented by the symbol set E. Theoretically an unlimited number of objects can be transported, it is possible to obtain a calculation result infinity; The calculation process is the initial state after a series of state transitions. Marking the success of the calculation rules cannot be used in the state, and, in the case of absence of the membrane dissipates. Figure 1 illustrate the calculation process, the following diagram is a communication $\mathrm{P}$ system contains four membranes.

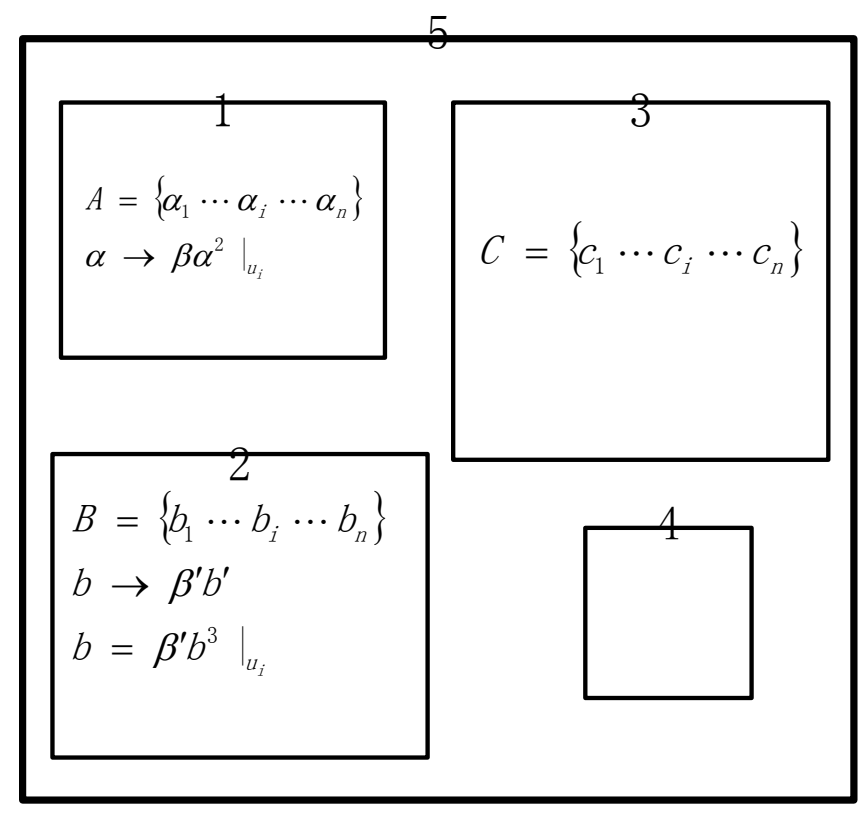

\section{Figure 1. Communication P System}

Figure 1 also shows the $\mathrm{P}$ system in the macro environment, the system includes four membranes, labels, respectively, 1, 2, 3 and 4. Labeled 5 is called the surface membrane, and membrane 4 does not contain other membrane are known as the basic membrane. Each membrane part of the region surrounded is called regional, contains the objects $A=\left\{a_{1} \cdots a_{i} \cdots a_{n}\right\}$ and the corresponding evolution rules. Just like any other computer device, computer program or an abstract model, $\mathrm{P}$ system from the initial state (the object that the multiple set) complete calculation through the use of membrane structure in the evolution of the rules of procedure coding. Figure 2 shows an example of the calculation is:

(1) in the initial state, the area defined in the membrane 1 contains a set of objects $A=\left\{a_{1} \cdots a_{i} \cdots a_{n}\right\}, a_{i}$ represents an object, membrane 2 contains a set of objects $B=\left\{b_{1} \cdots b_{i} \cdots b_{m}\right\} \quad$,membrane $3 \quad$ contains a set of objects $C=\left\{c_{1} \cdots c_{i} \cdots c_{v}\right\}$, membrane 4 is empty;

(2) membrane 1 there is a catalyst $\beta^{\prime}$, and on behalf of individual travel costs to membrane 2, represent congestion charges, the evolution rules $\left.a \rightarrow \beta^{\prime} a_{2}\right|_{u_{i}}$ in every step will send an object ${ }^{a_{i}}$ into the membrane 2 ; at the same time membrane 2 under the rules $b \rightarrow \beta^{\prime} b_{3}$ in the catalyst $\beta$ will send object $b_{i}$ continuously into the membrane 3 , until 
the number equal to a specified threshold in one area or reaches the evolution of generation.

\section{Communication P System Traffic Selection Algorithms (CPS)}

CPS iterative algorithm Step as follows [22]:

Step1: Initialize. Set operating parameters, such as maximum operating generation, the size of the object set, the number of variables, etc., without designated initialized population case, each membrane (area) given randomly initialized population.

Step2: membrane evolution. The process of evolution in the membrane, its rules can be used in any order, while the evolution of each membrane at the same time, given the parameters $u_{i}$ and travel costs parameters $\beta^{\prime}$ of each membrane.

Step3: According to the exchange rules, all membrane exchanges some of their objects.

Step4: If the exchange number of the object greater than saturation capacity ${ }_{a}$ in a time period, the algorithm terminates, re-adjust the parameters of ${ }^{u_{i}}$, return step 3 . This parameter of $u_{i}$ refers to the influence of the traveler's random perturbation (also referred to as congestion charging value), this parameter determines the traveler to say "yes" or "no" behavior, this parameter dynamically adjustable, will affect the evolution and development of the entire system.

Step5: the termination condition can be determined according to the actual situation, For example, when the value of the object to achieve the best pre-satisfactory results. A common pre-specified termination condition is to run the evolution of generation.

\section{Simulation Experiment}

Using a small road network as an example, seven nodes, including three starting point, one end point, eleven roads, and the road network structure are shown in Figure 2.

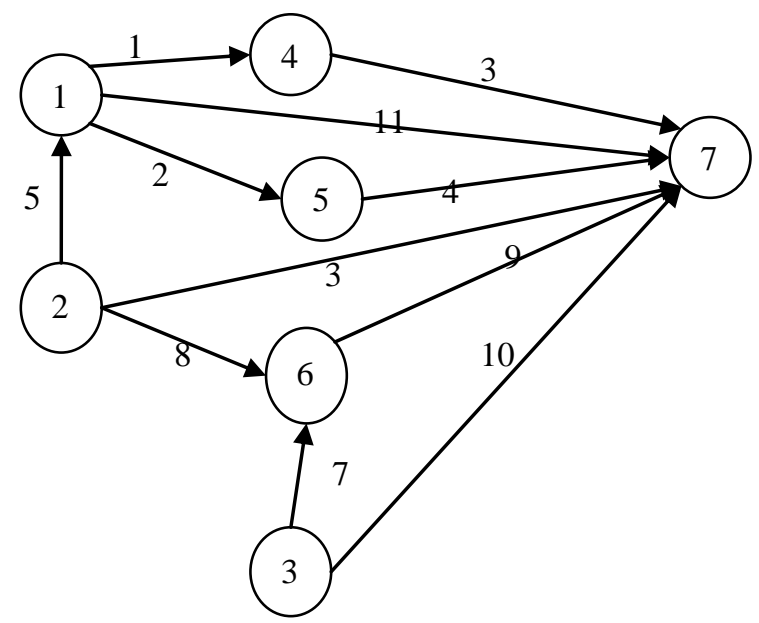

Figure 2. Seven Node Network Structure

Assuming that toll road charges, respectively, upper and lower limits for $0 \leq u_{a} \leq 10$, the use of BPR travel time function is expressed as

$$
t_{a}=t_{a}^{0}\left[1+\alpha\left(\frac{x_{a}}{c_{a}}\right)^{\beta}\right],
$$


Where, ${ }^{t} a$ is the roads $a$ on the free flow time; ${ }^{c}{ }_{a}$ is the roads capacity, $\alpha, \beta$ for the coefficient values were 0.15 and 4 .

The travel amount $N$ on road ${ }^{a}$ was calculated by using the formula (6), which is expressed as:

$$
N=E_{a}^{p}\left(x_{a}\right)=A\left(p, c_{a}\right) l_{a} e^{B\left(p, c_{a}\right) x_{a}},
$$

In the formula (6), $A\left(p, c_{a}\right)$ and $B\left(p, c_{a}\right) x_{a}$ are constants related to the probability $p_{\text {of making travel and capacity saturation }} c_{a}$ on roads $a . u_{i}$ is road congestion charging, the initial value was given randomly, network basic input parameters are showed in Table 1.

In the evolutionary process, in order to prevent too much the string to the same membrane, the limit maximum number of repetitions of the object population size of $1 / 3$ is adopted. Table 2 shows the calculated road congestion charges ${ }_{i}$ after a certain time evolution.

In order to compare the advantages and disadvantages algorithm, Congestion pricing model (CPS _ DTAM) based on P system were compared with congestion pricing model (PSO_ DTAM) based on particle swarm optimization under the same conditions. Some of the data was cited from literature [12], the results show that the CPS_ DTAM is much more accord to the actual traffic situation, the reason may be some travelers will change course or change the travel time after charging and considering the travelers' decision.

Table 1. Network Input Parameters

\begin{tabular}{llllllllllll}
\hline$a$ & 1 & 2 & 3 & 4 & 5 & 6 & 7 & 8 & 9 & 10 & 11 \\
\hline$u_{i}$ & 6 & 5 & 2 & 1 & 6 & 1 & 5 & 0 & 4 & 3 & 1 \\
$c_{a}$ & 200 & 200 & 200 & 100 & 100 & 100 & 150 & 150 & 200 & 200 & 200 \\
$N$ & 60 & 70 & 80 & 30 & 150 & 50 & 40 & 50 & 100 & 60 & 50 \\
\hline
\end{tabular}

Table 2. The Road Congestion Charges

\begin{tabular}{llllllllllll}
$a$ & 1 & 2 & 3 & 4 & 5 & 6 & 7 & 8 & 9 & 10 & 11 \\
\hline$u_{i}$ & 3.2 & 2.8 & 2.4 & 3.3 & 0 & 2 & 3.8 & 3 & 2 & 2.4 & 4 \\
\hline
\end{tabular}

Table 3. Comparison of Different Algorithms Model

\begin{tabular}{cccccc}
\hline & Fees & A flow path & B flow path & Total Flow & $\begin{array}{c}\text { The average vehicle } \\
\text { delay }\end{array}$ \\
\hline No charge & 0 & 3856 & 2608 & 6464 & 30.31 \\
\hline \multirow{3}{*}{ PSO_DTAM } & Rate 1 & 3457.3 & 2185.4 & 5642.7 & 23.33 \\
\cline { 2 - 6 } & Rate 2 & 3133.2 & 2098.2 & 5231.4 & 22.85 \\
\cline { 2 - 6 } & Rate 3 & 3033.8 & 2100.5 & 5134.3 & 22.03 \\
\hline \multirow{3}{*}{ CPS_DTAM } & Rate 1 & 3043.5 & 1974.2 & 5017.7 & 20.23 \\
\cline { 2 - 6 } & Rate 2 & 2884.2 & 1865.8 & 4750.0 & 18.64 \\
\cline { 2 - 6 } & Rate 3 & 2589.4 & 2018.5 & 4607.9 & 15.32 \\
\hline
\end{tabular}

Note: PSO_DTAM data references [12] 


\section{Conclusions}

In this paper, congestion road pricing model and its evolutionary algorithms were established based on $\mathrm{P}$ system. We selected a small network for example, with seven nodes, including three starting point, an end point 11 sections. After considering the congestion charge, the same sections of different charges will affect the traffic flow, as well as the level of charges will affect travelers make different choices. The simulation results verify the correctness and feasibility of congestion pricing model. This model and particle swarm optimization congestion pricing model (PSO_ DTAM) were compared under the same parameters; the results show that these pricing models consider the dynamic decision more in line with the real situation. So far, P systems in intelligent control, NP problems, has been part of intelligent medical applications, but it is still relatively preliminary, $\mathrm{P}$ system as a relatively new optimization algorithm, the researchers think through the combination with other intelligent algorithms, put forward various hybrid $\mathrm{P}$ system optimization methods, and promote the development of intelligent optimization algorithms, with extensive research value. P system is applied to calculate the congestion charge model is an attempt, providing a possible approach to related researchers.

\section{Acknowledgements}

This work was supported by China Postdoctoral Science Foundation (No.2013M542370) and by the Specialized Research Fund for the Doctoral Program of Higher Education of China (Grant No. 20136118120010) and by Scientific Research Program Funded by Shaanxi Provincial Education Department (Program No.2013JK1139).

\section{References}

[1] X. Wei, "Research on Traffic Assignment Model Considering Emission Effects and Solution Algorithm", Wuhan: Wuhan University of Technology, (2008).

[2] C. Hongjian, "Study on Optimization Mechanism and Means of Urban Traffic Structure", Xi'an: Chang'an University, (2010).

[3] W. Yan, "Thinking about road congestion charges levied", Industry and Technology Forum, vol. 10, no. 17, (2011), pp. 60-63.

[4] R. W. Rosenthal, "A class of games possessing pure-strategy Nash equilibria”, International Journal of Game Theory, vol. 2, no. 1, (1973), pp. 65-67.

[5] D. Levinson, "Micro-foundations of congestion and pricing: a game theory perspective", Transportation Research Part A, vol. 39, (2005), pp. 691-704.

[6] Z. Yimei and Y. K. fei, "Traffic Congestion Mechanism Analysis Based on Economic Theory", Journal of Tongji University (Natural Science), vol. 34, no. 3, (2006), pp. 359-362.

[7] X. Wei and J. Yuanhua, "Analysis of city road traffic congestion game", Traffic science \& Technology, vol. 2, (2006), pp. 80-82.

[8] L. Zhigang and S. Jinsheng, "Analysis of Urban Traffic Jam Based on game Theory”, City traffic, vol. 2, (2005), pp. 63-65.

[9] W. Bing and L. Linbo, "Evolution Dynamic Analysis of Traffic Congestion", China Journal of highway and transport, vol. 19, no. 3, (2006), pp. 106-110.

[10] C. Ying and L. Jun, "Study of urban road's Traffic Congestion Pricing Based on Cooperative game Theory", Operations Research and Management Science, vol. 22, no. 1, (2013), pp. 9-14.

[11] H. Nan and Z. Shengchuan, "Road Induced Traffic and Solutions to Relative Transportation Policies", Transportation Science and Technology, vol. 257, no. 2, (2013), pp. 143-146.

[12] Z. Chaihou and L. Weiming, "Research on Dynamic Traffic Assignment Model and Simulation Using Particle Swarm Optimization Algorithm", Science Technology and Engineering, vol. 8, no. 10.

[13] G. Paun, "Computing with membranes", Journal of Computer and System Sciences, vol. 1, no. 61, (2000), pp. 108-143.

[14] L. Huang, X. X. He and N. Wang, "systems based multi-objective optimization algorithm", Progress in Natural Science, vol. 17, no. 4, (2007), pp. 458-465.

[15] H. Liang, "Research on Membrane Computing Optimization Methods", Hangzhou: Zhejiang University, (2007).

[16] L. Pan and C. Martin-Vide, "Solving multidimensional 0-1knapsaek Problem by Systems with input and 
active membranes", Journal of Parallel and Distributed Computing, vol. 65, no. 12, (2005), pp. 15781584.

[17] T. Y. Nishida, "An Application of P System: A new Algorithm for NP-complete Optimization Problems", In: Proceeding of 8th world Multi- conference on Systems. Orlando: Cybernetics and Informatics, (2004), pp. 18-21.

[18] A. Miao, "Research on Some Arithmetic operations and Simulation of membrane computing model", Harbin: Harbin Institute of Technology, (2010).

[19] Z. Gexiang, "A Survey of Membrane Computing As a New Branch of Natural Computing”, Chinese Journal of computers, vol. 33, no. 2, (2010), pp. 208-214.

[20] A. Paun and G. Paun, "The Power of Communication: P Systems with Symport/antipart", New Generation Computing, vol. 20, no. 3, (2002), pp. 295-305.

[21] G. Zhang, M. Gheorghe and C. Wu, "A Quantum-Inspired Evolutionary Algorithm Based on P systems for Knapsack problem”, Fundamentals Informatics, vol. 87, no. 1, (2008), pp. 93-116.

[22] Z. Yanhua, L. Yaolin and N. Shuzhi, "Road Congestion Pricing Model Based on P System", icicta, , 2012 Fifth International Conference on Intelligent Computation Technology and Automation, (2012). pp. 295-298.

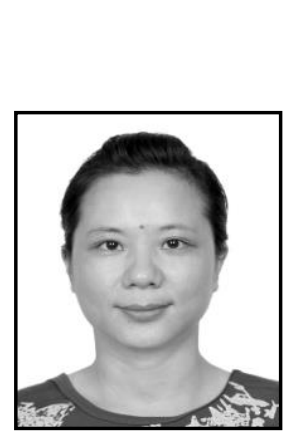

\section{Authors}

Zhong Yanhua, she is Software Engineering M.S., graduated from Computer College, Guangdong University of Technology, China. And research interests on intelligent algorithms and quantum computing. She is an associate professor of Jiangmen Polytechnic.

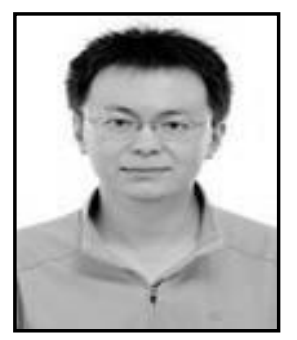

Wei Wei, he received his Ph.D. and M.S. degrees from Xi'an Jiaotong University in 2011 and 2005, respectively. Currently he is an assistant Professor at Xi'an University of Technology. His research interests include Wireless Networks and Wireless Sensor Networks Application, Mobile Computing, Distributed Computing, and Pervasive Computing.

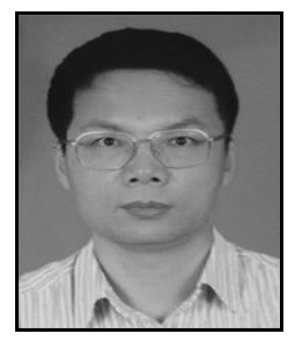

Liu Zhiyong, he received the Doctor degree in Information and Control Engineering, Xi'an Jiao Tong University, Xi'an, China, in 1989. He is currently a professor at Jiangmen Polytechnic. His main area of research interest is Intelligent Transportation. 